\title{
Ponderomotive forces in electrodynamics of moving media: The Minkowski and Abraham approaches
}

\author{
V.V. Nesterenkd* and A.V. Nesterenko \\ Bogoliubov Laboratory of Theoretical Physics, \\ Joint Institute for Nuclear Research, \\ 141980 Dubna, Russia
}

(Dated: October 8, 2018)

\begin{abstract}
In the general setting of the problem, the explicit compact formulae are derived for the ponderomotive forces in the macroscopic electrodynamics of moving media in the Minkowski and Abraham approaches. Taking account of the Minkowski constitutive relations and making use of a special representation for the Abraham energy-momentum tensor enable one to obtain a compact expression for the Abraham force in the case of arbitrary dependence of the medium velocity on spatial coordinates and the time and for nonstationary external electromagnetic field. We term the difference between the ponderomotive forces in the Abraham and Minkowski approaches as the Abraham force not only under consideration of media at rest but also in the case of moving media. The Lorentz force is found which is exerted by external electromagnetic field on the conduction current in a medium, the covariant Ohm law and the constitutive Minkowski relations being taken into account. The physical argumentation is traced for definition of the 4 -vector of the ponderomotive force as the 4-divergence of the energy-momentum tensor of electromagnetic field in a medium.
\end{abstract}

PACS numbers: 03.30.+p, 03.50.De, 41.20.-q

Keywords: Electrodynamics of moving media, Minkowski energy-momentum tensor, Abraham energymomentum tensor, ponderomotive forces

* E-mail: nestr@theor.jinr.ru 


\section{CONTENTS}

I. Introduction

II. Minkowski macroscopic electrodynamics of moving media

III. Ponderomotive forces and the energy-momentum tensor

IV. Ponderomotive forces in the Minkowski approach

V. Different representations of the Abraham energy-momentum tensor

VI. Ponderomotive forces in the Abraham approach

VII. Conclusion

A. Ponderomotive forces in vacuum

B. The explicit formula for vector $\mathfrak{W}$

\section{INTRODUCTION}

In macroscopic electrodynamics, there is no generally accepted definition of the ponderomotive forces. Usually this problem is bound up with the definition of the energy-momentum tensor of electromagnetic field in a medium, with presupposing the ponderomotive force being equal to the four-dimensional divergence of this tensor and with taking into account the Maxwell equations. Up to now the use of the theoretical argumentation did not allow one to define uniquely the energy-momentum tensor in medium. Therefore when analyzing the ponderomotive force, the working formulae are useful which are derived by making use of the different versions of this tensor. It is this problem that is considered in the present paper. We derive compact 3-dimensional vector formulae for ponderomotive forces in the case of moving media by making use of the electromagnetic energy-momentum tensor in the Minkowski form and in the Abraham form. These tensors proposed more than one century ago are the most popular and are often discussed [1 13$]$. In the framework of other approaches 
they are treated as distinctive "fulcra" (see, for example, Ref. [4]).

We do not touch the problem of determining the 'correct' energy-momentum tensor in macroscopic electrodynamics and the Abraham-Minkowski controversy. There is a large body of the literature on this subject, see, for example, the reviews [1, 2, 5-7] and references therein.

In macroscopic electrodynamics, the important role is played by the constitutive relations between field vectors. Only due to these relations the formal scheme of this theory acquires the physical content [8, Sec. 39]. We shall use the constitutive relations in the form derived by Minkowski. The values of the vectors $\mathbf{E}, \mathbf{H}, \mathbf{D}, \mathbf{B}$ and the velocity of the medium $\mathbf{v}, v<c$, which satisfy the constitutive relations will be refereed to as the physical configuration space $\bar{\Gamma}$ in contrast to the whole unrestricted configuration space $\Gamma$. In practical calculations aimed at the obtaining of the final physical results, one assumes that the constitutive relations will be eventually used. Therefore in deriving the formulae for the ponderomotive forces it makes sense to take into account, aside from the Maxwell equations, the constitutive relations too. Minkowski [9] and Abraham [10-12] proceeded just in this way. This simplifies essentially the ultimate formulae for moving media, however the realization of this task turns out to be rather complicated.

In the general case, the ponderomotive forces include those of two types, the Lorentz force experienced by free charges and currents, and the forces exerted by electromagnetic field on a medium. When deriving the Lorentz force from the divergence of the energymomentum tensor, the Maxwell equations are used. The remaining part of the divergence is transformed with allowance for the constitutive equations resulting in the formulae for the ponderomotive forces defined on $\bar{\Gamma}$.

As known, in the case of a medium at rest the Abraham energy-momentum tensor gives an additional, as compared with the Minkowski approach, term for the ponderomotive force, the so-called Abraham force. This force is different from zero only for the external field dependent on time. In the case of moving media the difference between the Abraham approach and Minkowski approach turns out to be more substantial because the Abraham energy-momentum tensor, in contrast to the Minkowski tensor, depends explicitly on the medium velocity. For practical use, it makes sense to derive such formulae for ponderomotive forces in which this distinction is shown clearly and the formulae themselves are simple as much as possible. Further we shall call the difference between the ponderomotive forces in 
the Abraham and Minkowski approaches as the Abraham force both in the case of media at rest and in the case of moving media.

The making use of a special representation for the Abraham energy-momentum tensor on $\bar{\Gamma}$, which is the most close, in structure, to the Minkowski tensor, will enable us to obtain a compact formula for the Abraham force in the case of moving media with an arbitrary dependence of the medium velocity on the coordinates and time and for the time dependent external electromagnetic field.

Before beginning the calculation of the ponderomotive forces, we consider the physical reasoning which is adduced in favour of defining these forces through the divergence of the energy-momentum tensor of electromagnetic field in medium. In particular, it will be shown that this argumentation is insufficient for defining this tensor uniquely.

The layout of the paper is as follows. In Sec. II, the Minkowski electrodynamics of moving media is briefly stated. In Sec. III, the physical reasoning is retraced which leads to the definition of the 4 -vector of the ponderomotive force in terms of the divergence of the electromagnetic energy-momentum tensor in medium. In Sec. IV, the formulae for the ponderomotive forces are derived on $\bar{\Gamma}$ in the framework of the Minkowski approach. The general setting of the problem is considered, namely, nonhomogeneous moving medium with arbitrary dependence of its velocity on coordinates and time and the nonstationary external electromagnetic field. In addition, the macroscopic Lorentz force is found here which is exerted by external electromagnetic field on the conduction current in a medium, the covariant Ohm law and the constitutive Minkowski relations being taken into account. In Sec. $\mathrm{V}$ the different representations of the Abraham energy-momentum tensor on $\bar{\Gamma}$ are discussed. In Sec.VI the formulae are derived for the ponderomotive forces in the Abraham approach. For that a special representation of the Abraham tensor is employed which is the most close, according to its structure, to the Minkowski energy-momentum tensor on $\bar{\Gamma}$. In Conclusion (Sec. VII) the obtained results are briefly summarized. In Appendix A in the framework of the electrodynamics in vacuum, the interrelation is traced between the ponderomotive forces (in the present case between the Lorentz force) and the energymomentum tensor of electromagnetic field in vacuum. In Appendix $\mathbb{B}$, the explicit formula is derived for the vector $\mathfrak{W}$ the making use of which enables one to represent, in a compact form, the ultimate formulae for the ponderomotive forces on $\bar{\Gamma}$.

We use the following notations. The coordinates $x_{\alpha}$ in the Minkowski space have a pure 
imaginary time component: $x_{\alpha}=\left(x_{1}, x_{2}, x_{3}, x_{4}=\mathrm{i} c t\right)=(\mathrm{x}, \mathrm{i} c t)$. The Greek indices take values $1,2,3,4$, whereas the Latin indices assume the values $x, y, z$ or $1,2,3$; the summation over the repeated indexes is understood in respective range. The unrationalized Gaussian units are used for the electromagnetic field and the notations generally accepted in macroscopic electrodynamics [13] are adopted.

\section{MINKOWSKI MACROSCOPIC ELECTRODYNAMICS OF MOVING MEDIA}

We shall consider the electromagnetic field in a material media in the framework of the Minkowski phenomenological electrodynamics [8, Secs. 33-35]. Here we give the basic equations defining this theory. The electromagnetic field in a medium is described by the macroscopic Maxwell equations

$$
\begin{gathered}
\operatorname{rot} \mathbf{E}+\frac{1}{c} \frac{\partial \mathbf{B}}{\partial t}=0, \quad \operatorname{div} \mathbf{B}=0, \\
\operatorname{rot} \mathbf{H}-\frac{1}{c} \frac{\partial \mathbf{D}}{\partial t}=\frac{4 \pi}{c} \mathbf{j}, \quad \operatorname{div} \mathbf{D}=4 \pi \rho .
\end{gathered}
$$

In a covariant form these equations read

$$
\begin{gathered}
\frac{\partial F_{\alpha \beta}}{\partial x_{\gamma}}+\frac{\partial F_{\beta \gamma}}{\partial x_{\alpha}}+\frac{\partial F_{\gamma \alpha}}{\partial x_{\beta}}=0, \\
\frac{\partial H_{\alpha \beta}}{\partial x_{\beta}}=\frac{4 \pi}{c} j_{\alpha} .
\end{gathered}
$$

Here $F_{\alpha \beta}$ and $H_{\alpha \beta}$ are two antisymmetric tensors introduced by Minkowski [8, Sec. 33], [13, $\S 76]$,

$$
\begin{gathered}
F_{i j}=\varepsilon_{i j k} B_{k}, \quad F_{4 j}=-F_{j 4}=\mathrm{i} E_{j} \\
H_{i j}=\varepsilon_{i j k} H_{k}, \quad H_{4 j}=-H_{j 4}=\mathrm{i} D_{j} .
\end{gathered}
$$

It is convenient to represent Eqs. (2.5) and (2.6) in a short notation, explicitly indicating the 3-dimensional vectors, which define the components of the antisymmetric tensors [9, 14, 15]:

$$
F_{\alpha \beta}(\mathbf{B} ;-\mathrm{i} \mathbf{E}), \quad H_{\alpha \beta}(\mathbf{H} ;-\mathrm{i} \mathbf{D}) .
$$

In the general case, the current density in nonhomogeneous Maxwell equations (2.2), (2.4)

$$
j_{\alpha}=(\mathbf{j}, \mathrm{i} c \rho)
$$


is the sum of two terms, the density of the conduction current and the density of convection current (see Eq. (2.22) and further). The total current density $j_{\alpha}$ satisfies the continuity equation

$$
\operatorname{div} \mathbf{j}+\frac{\partial \rho}{\partial t} \equiv \frac{\partial j_{\alpha}}{\partial x_{\alpha}}=0
$$

which entails the conservation of the charge. Equation (2.9) is the consistency condition of the Maxwell equations (2.2), (2.4).

The Maxwell equations (2.1)-(2.4) and continuity equation (2.9) constitute a formal mathematical scheme, which is not filled with the physical content yet. Indeed, the number of these equations in the material medium and in vacuum is the same, whereas the number of the field functions in a medium is two times as large as compared with vacuum. Further, the equations in question do not contain the material characteristics of medium. These problems are removed by introducing the constitutive relations which play an important role in macroscopic electrodynamics.

We shall consider a linear isotropic medium without temporal and spatial dispersion with the constitutive relations

$$
\mathbf{D}=\varepsilon \mathbf{E}, \quad \mathbf{B}=\mu \mathbf{H}
$$

(medium at rest). In order to generalize (2.10) to moving medium, Minkowski introduced the Lorentz vectors $E_{\alpha}, D_{\alpha}$ which in the co-moving reference frame, $K^{\prime}$, where the medium is at rest $\left(\mathbf{v}^{\prime}=0\right)$, have the components

$$
E_{\alpha}^{\prime}=\left(\mathbf{E}^{\prime}, 0\right), \quad D_{\alpha}^{\prime}=\left(\mathbf{D}^{\prime}, 0\right), \quad H_{\alpha}^{\prime}=\left(\mathbf{H}^{\prime}, 0\right), \quad B_{\alpha}^{\prime}=\left(\mathbf{B}^{\prime}, 0\right) .
$$

The prime implies that the respective quantity is considered in the co-moving frame. Obviously conditions (2.11) determine the vectors $E_{\alpha}, D_{\alpha}$ and $H_{\alpha}, B_{\alpha}$ uniquely. Minkowski [9] constructed for these vectors the explicit formulae (see also [5, 15, 16])

$$
\begin{gathered}
E_{\alpha}=F_{\alpha \beta} u_{\beta}=\gamma\{\mathbf{E}+[\mathbf{q B}], \mathrm{i}(\mathbf{q} \mathbf{E})\}, \quad D_{\alpha}=H_{\alpha \beta} u_{\beta}=\gamma\{\mathbf{D}+[\mathbf{q H}], \mathrm{i}(\mathbf{q} \mathbf{D})\}, \\
H_{\alpha}=-\mathrm{i} H_{\alpha \beta}^{*} u_{\beta}=\gamma\{\mathbf{H}-[\mathbf{q D}], \mathrm{i}(\mathbf{q} \mathbf{H})\}, \quad B_{\alpha}=-\mathrm{i} F_{\alpha \beta}^{*} u_{\beta}=\gamma\{\mathbf{B}-[\mathbf{q} \mathbf{E}], \mathrm{i}(\mathbf{q B})\} .
\end{gathered}
$$

Here $u_{\alpha}$ is the 4 -velocity of the medium

$$
u_{\alpha}=\gamma\{\mathbf{q}, \mathrm{i}\}, \quad \mathbf{q}=\mathbf{v} / c, \quad \gamma^{-1}=\sqrt{1-\mathbf{q}^{2}}, \quad u_{\alpha} u_{\alpha}=-1, \quad \alpha=1,2,3,4,
$$

and $F_{\alpha \beta}^{*}$ and $H_{\alpha \beta}^{*}$ are the tensors which are dual to the tensors $F_{\alpha \beta}$ and $H_{\alpha \beta}$ respectively:

$$
F_{\alpha \beta}^{*}=\frac{1}{2} \varepsilon_{\alpha \beta \gamma \delta} F_{\gamma \delta}, \quad H_{\alpha \beta}^{*}=\frac{1}{2} \varepsilon_{\alpha \beta \gamma \delta} H_{\gamma \delta}, \quad \varepsilon_{1234}=+1
$$


By making use of notation (2.7), we can write

$$
-\mathrm{i} F_{\alpha \beta}^{*}(-\mathbf{E} ;-\mathrm{iB}) ; \quad-\mathrm{i} H_{\alpha \beta}^{*}(-\mathbf{D} ;-\mathrm{i} \mathbf{H})
$$

From definitions (2.12) and (2.13) it follows that the vectors $E_{\alpha}, D_{\alpha}$ and $H_{\alpha}, B_{\alpha}$ are orthogonal to the medium velocity $u_{\alpha}$

$$
E_{\alpha} u_{\alpha}=0, \quad D_{\alpha} u_{\alpha}=0, \quad H_{\alpha} u_{\alpha}=0, \quad B_{\alpha} u_{\alpha}=0
$$

and at $\mathbf{q}=0$ satisfy condition (2.11) . In the covariant form the constitutive relations (2.10) are written as

$$
\begin{gathered}
D_{\alpha}=\varepsilon E_{\alpha}, \\
B_{\alpha}=\mu H_{\alpha}
\end{gathered}
$$

or in the 3-dimensional vector notation [8, Sec. 33], [13, § 76]

$$
\begin{aligned}
& \mathbf{D}+[\mathbf{q} \mathbf{H}]=\varepsilon(\mathbf{E}+[\mathbf{q} \mathbf{B}]), \\
& \mathbf{B}-[\mathbf{q} \mathbf{E}]=\mu(\mathbf{H}-[\mathbf{q} \mathbf{D}])
\end{aligned}
$$

As noted in Introduction, the values of the field variables $\mathbf{E}, \mathbf{H}, \mathbf{D}, \mathbf{B}$ and the velocity of a medium 1 v $\mathbf{v} v<c$, which satisfy the constitutive Minkowski relations (2.18) -(2.21), will be referred to as the physical configuration space $\bar{\Gamma}[3]$.

The material relation for the current density (2.8) is derived in a similar way [9]. As noted above, in the general case the current density $j_{\mu}$ is the sum of the density of conduction current and the density of convection current,

$$
j_{\mu}=j_{\mu}^{\text {cond }}+j_{\mu}^{\text {conv }}
$$

In the co-moving frame $K^{\prime}$, where the medium is at rest $(\mathbf{v}=0)$, these densities, by definition, have the components [17, § 60]

$$
\begin{gathered}
\left(j_{\mu}^{\text {cond }}\right)^{\prime}=\left(\mathbf{j}_{\text {cond }}^{\prime}, 0\right), \\
\left(j_{\mu}^{\text {conv }}\right)^{\prime}=\left(\mathbf{0}, \mathrm{i} c \rho_{\text {conv }}^{\prime}\right) .
\end{gathered}
$$

\footnotetext{
${ }^{1}$ We do not consider the case when the medium velocity $\mathbf{v}$ is equal to the phase velocity of light in the given medium, $v^{2}=c^{2} /(\varepsilon \mu)$. For this value of $v$ the equations (2.20) and (2.21) cannot be resolved with respect to $\mathbf{D}$ and $\mathbf{B}$ (see [8, Sec. 33]).
} 
For simplicity, we introduce the notation [17, 18]

$$
\mathbf{j}_{\text {cond }}^{\prime}=\mathbf{j}^{0}, \quad \rho_{\text {conv }}^{\prime}=\rho^{0} .
$$

In the rest frame, we have for the total current (2.22)

$$
j_{\mu}^{\prime}=\left(j_{\mu}^{\text {cond }}\right)^{\prime}+\left(j_{\mu}^{\text {conv }}\right)^{\prime}=\left(\mathbf{j}^{0}, \mathrm{i} c \rho^{0}\right) .
$$

By making use of the Lorentz transformations the total current (2.22), in an arbitrary inertial reference frame $K$, can be found through its components (2.26) in the rest frame. We accomplish this transformation separately for the conduction current (2.23) and for the convection current (2.24). In the first case we have

$$
j_{\mu}^{\text {cond }}=\left(\mathbf{j}_{\text {cond }}, \mathrm{i} c \rho_{\text {cond }}\right)
$$

where

$$
\begin{aligned}
\mathbf{j}_{\text {cond }} & =\mathbf{j}^{0}+\frac{\mathbf{v}}{v^{2}}(\gamma-1)\left(\mathbf{v} \mathbf{j}^{0}\right), \\
c \rho_{\text {cond }} & =\frac{\gamma}{c}\left(\mathbf{v} \mathbf{j}^{0}\right)=\frac{1}{c}\left(\mathbf{v} \mathbf{j}_{\text {cond }}\right) .
\end{aligned}
$$

Here we have employed the Lorentz transformation for the case, when the velocity $\mathbf{v}$ of the co-moving frame $K^{\prime}$ relative to the inertial reference frame $K$ has arbitrary orientation [19], [8, Sec. 4], [20], [5, Chap. IV]. The spatial coordinate axes in $K$ and $K^{\prime}$ are parallel. Taking into account the explicit form of the conduction current $\mathbf{j}_{\text {cond }}(2.28)$, one can easily be convinced that in Eq. (2.29) the product $\gamma \mathbf{j}^{0}$ may be substituted by $\mathbf{j}_{\text {cond }}$.

Application of the Lorentz transformation specified above to the 4-vector (2.24) yields

$$
j_{\mu}^{\text {conv }}=\gamma\left(c \rho^{0} \frac{\mathbf{v}}{c}, \mathrm{i} c \rho^{0}\right)=\left(\rho_{\text {conv }} \mathbf{v}, \mathrm{i} c \rho_{\text {conv }}\right)=c \rho^{0} u_{\mu}
$$

where $\rho_{\text {conv }}=\gamma \rho^{0}$ is the density of the convection charge in an arbitrary inertial reference frame, $\rho_{\text {conv }} \mathbf{v}=\mathbf{j}_{\text {conv }}$ is the density of the convection current in this system, and $u_{\mu}$ is the 4 -velocity of the medium (2.14). Multiplying (2.30) by $u_{\mu}$, we obtain

$$
u_{\mu} j_{\mu}^{\text {conv }}=-c \rho^{0} .
$$

On account of condition (2.23) the conduction current is orthogonal to the 4 -velocity of the medium

$$
u_{\mu} j_{\mu}^{\mathrm{conv}}=0,
$$


therefore the convection current in Eq. (2.31) can be substituted by the total current

$$
u_{\mu} j_{\mu}=-\rho^{0}
$$

Now Eq. (2.30) for convection current can be rewritten in the following way

$$
j_{\mu}^{\mathrm{conv}}=-\left(u_{\nu} j_{\nu}\right) u_{\mu}
$$

Substituting (2.34) in (2.22), we obtain

$$
j_{\mu}^{\text {cond }}=j_{\mu}-j_{\mu}^{\text {cond }}=j_{\mu}+\left(u_{\nu} j_{\nu}\right) u_{\mu}
$$

Obviously the conduction current defined in this way satisfies condition (2.23).

The derived formulae enable us to deduce the material relation for current [9]. In the co-moving frame the Ohm law reads

$$
\mathbf{j}_{\text {cond }}^{\prime}=\lambda \mathbf{E}^{\prime}
$$

where $\lambda$ is the conductivity of the medium.

Evidently, the covariant extension of this formula is given by

$$
j_{\mu}^{\text {cond }}=\lambda E_{\mu}
$$

where the Lorentz vector $E_{\mu}$ is defined in (2.12).

Using Eqs. (2.12) and (2.23) one can easily check that in the rest frame $K^{\prime}$ Eq. (2.37) assumes the form (2.36). The covariant Ohm law (2.37) can be rewritten by making use of only the total current (2.35)

$$
j_{\mu}+\left(j_{\nu} u_{\nu}\right) u_{\mu}=\lambda E_{\mu}
$$

Substituting in Eq. (2.37) the explicit expression for the Lorentz vector $E_{\mu}$, we obtain

$$
\begin{aligned}
j_{\mu}^{\text {cond }} & =\left(\mathbf{j}_{\text {cond }}, i \mathrm{i} c \rho_{\text {cond }}\right)=\lambda E_{\mu} \\
& =\lambda \gamma\{\mathbf{E}+[\mathbf{q} \mathbf{B}], \mathrm{i}(\mathbf{q} \mathbf{E})\}, \\
\mathbf{j}_{\text {cond }} & =\lambda \gamma(\mathbf{E}+[\mathbf{q B}]), \\
c \rho_{\text {cond }} & =\lambda \gamma(\mathbf{q E})=\left(\mathbf{q} \mathbf{j}_{\text {cond }}\right) .
\end{aligned}
$$

Equation (2.41) was derived previously by making use of the Lorentz transformation (see Eq. (2.29) ). 
In the sequel we shall also use the covariant generalization of the 3-dimensional Poynting vector

$$
\mathbf{S}^{\mathrm{P}}=\frac{c}{4 \pi}[\mathbf{E H}] .
$$

This generalization was constructed by Minkowski [9, pp. 34,35] with the name Ruhstrahlvektor

$$
\Omega_{\mu}=-\mathrm{i} \varepsilon_{\mu \alpha \beta \gamma} E_{\alpha} H_{\beta} u_{\gamma} .
$$

One can easily be convinced that in the co-moving reference frame, where $u_{\alpha}=(0,0,0, \mathrm{i})$, the vector (2.43) has the components

$$
\Omega_{\mu}^{\prime}=\left(\left[\mathbf{E}^{\prime} \mathbf{H}^{\prime}\right], 0\right) .
$$

For simplicity we shall call the vector $\Omega_{\mu}$ by Minkowski vector.

We shall also use the 4 -vector $\widetilde{\Omega}_{\mu}$, which is obtained from the Minkowski vector $\Omega_{\mu}(2.43)$ by the substitution

$$
\mathrm{E} \rightarrow \mathrm{D}, \quad \mathrm{H} \rightarrow \mathrm{B}, \quad \mathrm{D} \rightarrow \mathrm{E}, \quad \mathrm{B} \rightarrow \mathbf{H}
$$

On the analogy of (2.43) the vector $\widetilde{\Omega}_{\mu}$ is defined by the formula

$$
\widetilde{\Omega}_{\mu}=-\mathrm{i} \varepsilon_{\mu \alpha \beta \gamma} D_{\alpha} B_{\beta} u_{\gamma} .
$$

In the co-moving reference frame the 4 -vector $\widetilde{\Omega}_{\mu}$ has the components

$$
\widetilde{\Omega}_{\mu}^{\prime}=\left(\left[\mathbf{D}^{\prime} \mathbf{B}^{\prime}\right], 0\right) .
$$

Obviously the vectors $\Omega_{\mu}$ and $\widetilde{\Omega}_{\mu}$ satisfy the condition

$$
\Omega_{\mu} u_{\mu}=0, \quad \widetilde{\Omega}_{\mu} u_{\mu}=0 .
$$

Closing this Section, one can say briefly that the constitutive relations take into account the influence of the media on the electromagnetic field.2 In order to be complete, the macroscopic electrodynamics should be able to predict the action of the external field on the media, because the electromagnetic field itself is manifested, on the physical level, only through this action, or more precisely through ponderomotive forces, which are experienced by charges, currents, and media 3

${ }^{2}$ Media play the role of a given "background" for the dynamics of electromagnetic field, i.e., it is supposed that the material characteristics of the media, $\varepsilon$ and $\mu$, do not depend on the external electromagnetic field. This specifies, in particular, the range of applicability of the macroscopic electrodynamics, namely, external fields should be much less than the intermolecular fields which determine the electric and magnetic characteristics of media, $\varepsilon$ and $\mu$. If such dependence is allowed, then it implies that the medium under consideration is nonlinear.

${ }^{3}$ It is convenient for us to single out macroscopic charges and currents from the material medium. 


\section{PONDEROMOTIVE FORCES AND THE ENERGY-MOMENTUM TENSOR}

In macroscopic electrodynamics [13], it is generally accepted the point of view, formulated first by Lorentz, according to which the macroscopic equations of electromagnetic field inside a medium should be obtained as a result of the averaging, over the physically infinitesimal volume, of the corresponding microscopic equations in vacuum with allowance for the pointlike charges and currents forming a medium. In this approach, all macroscopic forces which are exerted by external electromagnetic field on macroscopic charges and currents, are the result of averaging of the microscopic Lorentz forces acting in vacuum on microscopic charges $\rho_{\text {micro }}$ and $\mathbf{j}_{\text {micro }}$ :

$$
\overline{\mathbf{f}_{\text {micro }}^{\mathrm{L}}}=\overline{\rho_{\text {micro }} \mathbf{e}}+\frac{1}{c} \overline{\left[\mathbf{j}_{\text {micro }} \mathbf{h}\right]} \text {. }
$$

A line just above the letter denotes the aforesaid averaging; $\mathbf{e}$ and $\mathbf{h}$ are the strengthes of microscopic electric and magnetic fields, respectively. Microscopic fields include intermolecular fields and external field. The microscopic charges and currents are usually divided into bound and free. It is natural to carry out this separation in the rest frame $K^{\prime}$ :

$$
\begin{aligned}
& \rho_{\text {micro }}^{\prime}=\rho_{\text {free }}^{\prime}+\rho_{\mathrm{b}}^{\prime}, \\
& \mathbf{j}_{\text {micro }}^{\prime}=\mathbf{j}_{\text {free }}^{\prime}+\mathbf{j}_{\mathrm{b}}^{\prime} .
\end{aligned}
$$

The averaging in Eqs. (3.2) and (3.3) yields evidently the following result:

$$
\begin{aligned}
& \overline{\rho_{\text {micro }}^{\prime}}=\overline{\rho_{\text {free }}^{\prime}}+\overline{\rho_{\mathrm{b}}^{\prime}}=0+\rho_{\text {conv }}^{\prime}=\rho^{0}, \\
& \overline{\mathbf{j}_{\text {micro }}^{\prime}}=\overline{\mathbf{j}_{\text {free }}^{\prime}}+\overline{\mathbf{j}_{\mathrm{b}}^{\prime}}=\overline{\mathbf{j}_{\text {free }}^{\prime}}+\mathbf{0}=\overline{\mathbf{j}_{\text {cond }}^{\prime}}=\mathbf{j}^{0},
\end{aligned}
$$

where the quantities $\rho^{0}$ and $\mathbf{j}^{0}$ were introduced before in defining the macroscopic convection current $j_{\mu}^{\text {conv }}$ and macroscopic conduction current $j_{\mu}^{\text {cond }}$ (see Eqs. (2.22) $-(2.26)$ ).

Let us assume further that the separate multipliers in Eq. (3.1) can be averaged independently 4 Taking into account definitions $\overline{\mathbf{e}}=\mathbf{E}, \overline{\mathbf{h}}=\mathbf{B}$ and Eqs. (3.4), (3.5), we obtain only the macroscopic Lorentz force applied to macroscopic charges and currents in the co-moving frame

$$
\left(\mathbf{f}_{\text {macro }}^{\mathrm{L}}\right)^{\prime}=\overline{\left(\mathbf{f}_{\text {micro }}^{\mathrm{L}}\right)^{\prime}}=\rho^{0} \mathbf{E}^{\prime}+\frac{1}{c}\left[\mathbf{j}^{\mathbf{0}} \mathbf{B}^{\prime}\right]
$$

\footnotetext{
4 This averaging does not pretend to a completeness and rigorous consideration being illustrative only.
} 
The covariant generalization of the macroscopic Lorentz force (3.6) is apparently given by

$$
\left(f_{\alpha}^{\mathrm{L}}\right)_{\text {macro }}=\frac{1}{c} F_{\alpha \beta} j_{\beta}=\left\{\rho \mathbf{E}+\frac{1}{c}[\mathbf{j B}], \mathrm{i}(\mathbf{q E})\right\},
$$

where the current $j_{\alpha}=\{\mathbf{j}$, ic $\rho\}$ is the sum of the convection current and conduction current (see Eqs. (2.22), (2.27), (2.30)).

At the end of Sec. IV, the macroscopic Lorentz force will be derived separately for the convection current and for the conduction current; in the latter case we will take into account the covariant generalization of the Ohm law (2.37) and Minkowski constitutive relations.

Thus the simple "naive" averaging of the microscopic Lorentz forces does not grasp the mechanical forces exerted by external electromagnetic field on the medium. More sophisticated averaging procedures require specification of the microscopic (molecular or atomic) structure of the medium and the results of such averaging turn out to be dependent on the chosen microscopic model (see, for example, [5] $]$ ).

Taking into account all of this, one has to follow indirect way.

When the dielectrid 5 is placed in an external electrostatic field $\mathbf{E}$, the volume density of the respective ponderomotive forces $\mathbf{f}^{(\mathrm{e})}$ is given by the formula [21 23$]$

$$
\mathbf{f}_{\text {stat }}^{(\mathrm{e})}=\rho \mathbf{E}-\frac{1}{8 \pi} E^{2} \boldsymbol{\nabla} \varepsilon+\frac{1}{8 \pi} \boldsymbol{\nabla}\left(E^{2} \frac{\partial \varepsilon}{\partial \tau} \tau\right),
$$

where $\rho$ is the macroscopic charge density (see the second equation in (2.2) ), $\tau$ is the medium density. This formula is derived by calculating the work accomplished by the electromagnetic field in dielectric by virtual displacement of the medium [21 23].

Fully similar one can derive the ponderomotive force exerted by the static magnetic field on the magnetic [21 23$]$

$$
\mathbf{f}_{\mathrm{stat}}^{(\mathrm{m})}=\frac{1}{c}[\mathbf{j} \mathbf{B}]-\frac{1}{8 \pi} H^{2} \boldsymbol{\nabla} \mu+\frac{1}{8 \pi} \boldsymbol{\nabla}\left(H^{2} \frac{\partial \mu}{\partial \tau} \tau\right) .
$$

Here $\mathbf{j}$ is the macroscopic current density (see the first equation in (2.2) ).

The last terms in Eqs. (3.8) and (3.9) are the volume densities of electric striction and magnetic striction forces, that do not contribute to the net force applied to a macroscopic body. Therefore we will disregard striction forces in what follows and assume that the volume density of the ponderomotive force for static field is defined by

$$
\mathbf{f}_{\text {stat }}=\mathbf{f}_{\text {stat }}^{(\mathrm{e})}+\mathbf{f}_{\text {stat }}^{(\mathrm{m})}=\rho \mathbf{E}+\frac{1}{c}[\mathbf{j} \mathbf{B}]-\frac{1}{8 \pi} E^{2} \boldsymbol{\nabla} \varepsilon-\frac{1}{8 \pi} H^{2} \nabla \mu .
$$

\footnotetext{
${ }^{5}$ Until the end of this Section, the medium is considered to be at rest.
} 
The first two terms on the right hand side of Eq. (3.10) is the macroscopic Lorentz force (3.7), exerted on charges and currents. The last two terms on the right hand side of (3.10) define the ponderomotive forces which are exerted by external electrostatic field $\mathbf{E}$ and by external magnetic field $\mathbf{H}$ on the medium. Ultimately we are interested just in these forces, but in intermediate calculations it is convenient for us to retain in (3.10) the Lorentz force (3.7) too.

In the textbooks [21 23] it is proved that the ponderomotive force (3.10) caused by static electromagnetic field can be reduced to the Maxwell tensions

$$
\mathbf{f}_{\text {stat }}=\boldsymbol{\sigma}, \quad \boldsymbol{\sigma}=\left(\sigma_{1}, \sigma_{2}, \sigma_{3}\right), \quad \sigma_{i} \equiv \frac{\partial \sigma_{i k}}{\partial x_{k}},
$$

where $\sigma_{i k}$ is the Maxwell stress tensor of electromagnetic field in medium

$$
4 \pi \sigma_{i k}=E_{i} D_{k}+H_{i} B_{k}-\frac{\delta_{i k}}{2}(\mathbf{E D}+\mathbf{H B}) .
$$

In order to verify Eq. (3.11), one has to substitute Eq. (3.12) in (3.11), take into account the Maxwell equations (2.1)-(2.2) with $\dot{\mathbf{D}}=0, \dot{\mathbf{B}}=0$, and make use of the constitutive equations (2.10). As a result Eq. (3.11) reduces to (3.10).

A consistent, physically motivated derivation of the formula for ponderomotive forces in macroscopic electrodynamics is restricted to the static external fields. To extend the obtained expression to the case of time dependent fields and cast it into explicitly covariant form one has to resort to some assumptions and use the analogy with electrodynamics in vacuum 6

Following this way, we assume preliminary that the density of ponderomotive force, $\mathbf{f}$, equals $\boldsymbol{\sigma}$ also in the case of time-dependent external electromagnetic field. We substitute again (3.12) into (3.11) and take advantage of the complete Maxwell equations (2.1) $-(2.2)$ and the constitutive relations (2.10). This yields

$$
\mathbf{f}=\boldsymbol{\sigma}=\rho \mathbf{E}+\frac{1}{c}[\mathbf{j} \mathbf{B}]-\frac{1}{8 \pi} E^{2} \boldsymbol{\nabla} \varepsilon-\frac{1}{8 \pi} H^{2} \boldsymbol{\nabla} \mu+\frac{\partial}{\partial t}\left(\frac{1}{4 \pi c}[\mathbf{D B}]\right) .
$$

The obtained formula contains an additional, in comparison with (3.10), term

$$
\frac{\partial}{\partial t}\left(\frac{1}{4 \pi c}[\mathbf{D B}]\right)
$$

${ }^{6}$ Difficulties emerged in deriving the ponderomotive forces exerted on a medium and caused by timedependent external electromagnetic field are substantiated. The point is the static electric and magnetic fields can be, in principle, treated proceeding from the conception of action at a distance (instantaneous transmission of interaction). In contrast to this, the time-dependent fields result in a finite velocity of propagation of electromagnetic interaction. Here the idea of a short-range interaction is adopted, which 13

is based on the notion of a field playing the role of a physical carrier of interaction. 
caused by the time-dependence of electromagnetic field. In the case of vacuum the right-hand side of (3.13) should obviously contain only the Lorentz force (3.6).

It is instructive to retrace in what way the additional term (3.14) vanishes in vacuum. In this case formula (3.14) acquires the form

$$
\frac{\partial}{\partial t}\left(\frac{1}{4 \pi c}[\mathbf{E H}]\right) .
$$

In order to elucidate this question we take advantage of the following statement which is proved in electrodynamics in vacuum (see the Appendix A and also [8, § 30], [14, § 33], [24]): the Lorentz force (3.6), exerted on charges and currents in vacuum, can be represented in the following form:

$$
\mathbf{f}_{\mathrm{vac}}^{L}=\boldsymbol{\sigma}_{\mathrm{vac}}-\dot{\mathrm{g}}_{\mathrm{vac}} \text {. }
$$

Here $\boldsymbol{\sigma}_{\text {vac }}$ is $\boldsymbol{\sigma}$ calculated in vacuum, i.e., setting $\mathbf{D}=\mathbf{E}$ and $\mathbf{B}=\mathbf{H}$ (see Eqs. (3.11), (3.12) ), and $\mathbf{g}_{\mathrm{vac}}$ is the momentum density of electromagnetic field in vacuum

$$
\mathbf{g}_{\mathrm{vac}}=\frac{1}{4 \pi c}[\mathbf{E H}] \text {. }
$$

When calculating the right-hand side of Eq. (3.16), the complete Maxwell equations in vacuum (2.1) $-(2.2)$ should be taken into account (see the Appendix A).

In view of all this, the volume density of ponderomotive force, Eq. (3.13), exerted by the time-dependent external electromagnetic field on a medium, is generalized in the following way:

$$
\mathbf{f}=\boldsymbol{\sigma}-\dot{\mathrm{g}}
$$

where $\mathbf{g}$ is the linear momentum density of electromagnetic field in medium.

Unfortunately there is no generally accepted expression for the electromagnetic momentum density in a medium g. In this field, fruitless argument is already going on more a century. In most cases, the attention is payed to the definitions of $\mathbf{g}$ proposed by Minkowski and by Abraham in their time [8, Sec. 35]. It is these versions that will be considered in the present paper.

\section{PONDEROMOTIVE FORCES IN THE MINKOWSKI APPROACH}

Minkowski [9] defined electromagnetic momentum density in a medium by the formula

$$
\mathbf{g}^{\mathrm{M}}=\frac{1}{4 \pi c}[\mathbf{D B}] \text {. }
$$


This choice leads, in particular, to cancellation of the additional term (3.14) in Eq. (3.18). As a result, Eq. (3.18) defining the ponderomotive force density in the case of time-dependent electromagnetic field, coincides, after expanding $\boldsymbol{\sigma}$ and $\dot{\mathrm{g}}$, with (3.10) which defines this force for static external field

$$
\begin{aligned}
\left.\mathbf{f}^{\mathrm{M}}\right|_{\mathbf{v}=0} & =\boldsymbol{\sigma}-\dot{\mathbf{g}}^{\mathrm{M}} \\
& =\rho \mathbf{E}+\frac{1}{c}[\mathbf{j} \mathbf{B}]-\frac{1}{8 \pi} \mathbf{E}^{2} \boldsymbol{\nabla} \varepsilon-\frac{1}{8 \pi} \mathbf{H}^{2} \boldsymbol{\nabla} \mu=\mathbf{f}_{\text {stat }} .
\end{aligned}
$$

It will be recalled that a medium at rest is considered and in deriving Eq. (4.3) constitutive relations (2.10) have been used. Hence Eq. (4.3) is defined on $\bar{\Gamma}$.

In order to recast Eq. (4.2) into covariant form one has obviously to extend the Maxwell 3-dimensional stress tensor (3.12) to the 4-dimensional stress-energy-momentum tensor. Minkowski [9] defined the energy-momentum tensor of electromagnetic field in a medium in the following form:

$$
T_{\alpha \beta}^{\mathrm{M}}=\frac{1}{4 \pi}\left(F_{\alpha \gamma} H_{\gamma \beta}-\frac{\delta_{\alpha \beta}}{4} F_{\gamma \nu} H_{\nu \gamma}\right) .
$$

By analogy with electrodynamics in vacuum (see Eq. (A4)), formula (4.2), in a covariant form, is written as

$$
f_{\mu}^{\mathrm{M}}=\frac{\partial T_{\mu \nu}^{\mathrm{M}}}{\partial x_{\nu}} .
$$

The individual components of the tensor $T_{\alpha \beta}^{\mathrm{M}}$, defined in (4.4), are given by the formulae

$$
T_{\alpha \beta}^{\mathrm{M}}=\left(\begin{array}{cc}
\sigma_{i j}^{\mathrm{M}} & -\mathrm{i} c \mathbf{g}^{\mathrm{M}} \\
-\frac{\mathrm{i}}{c} \mathbf{S}^{\mathrm{M}} & w^{\mathrm{M}}
\end{array}\right),
$$

where

$$
\begin{gathered}
\sigma_{i j}^{\mathrm{M}}=\frac{1}{4 \pi}\left\{E_{i} D_{j}+H_{i} B_{j}-\frac{\delta_{i j}}{2}(\mathbf{E D}+\mathbf{H B})\right\} \\
\frac{1}{c} \mathbf{S}^{\mathrm{M}}=\frac{1}{c} \mathbf{S}^{\mathrm{P}}=\frac{1}{4 \pi}[\mathbf{E H}], \quad c \mathbf{g}^{\mathrm{M}}=\frac{1}{4 \pi}[\mathbf{D B}] \\
w^{\mathrm{M}}=\frac{1}{8 \pi}(\mathbf{E D}+\mathbf{H B}) .
\end{gathered}
$$

The Minkowski stress tensor (4.7) coincides with the Maxwell expression (4.7), the density of the energy current in the Minkowski energy-momentum tensor (4.8) is given by the Poynting vector $\mathbf{S}^{\mathrm{P}}$ (see Eq. (2.42)), and the momentum density $\mathbf{g}^{\mathrm{M}}$ in (4.8) reproduces Eq. (4.1).

By definition, Eq. (4.4) determines the Minkowski energy-momentum tensor both for media at rest and for moving media. The velocity of a medium, $\mathbf{v}$, does not enter into Eq. (4.4). The Minkowski tensor (4.4) is not symmetric $T_{\alpha \beta}^{\mathrm{M}} \neq T_{\beta \alpha}^{\mathrm{M}}$, its trace vanishes $T_{\alpha \alpha}^{\mathrm{M}}=0$. 
Now we turn to calculating the explicit covariant formula for ponderomotive force by making use of the covariant formula (4.5). For simplicity sake we introduce the notation

$$
\frac{\partial \varphi}{\partial x_{\nu}} \equiv \varphi_{, \nu}
$$

On substituting (4.4) into (4.5), we get

$$
\begin{aligned}
4 \pi T_{\alpha \beta, \beta}^{\mathrm{M}} & =F_{\alpha \gamma, \beta} H_{\gamma \beta}+F_{\alpha \gamma} H_{\gamma \beta, \beta}-\frac{\delta_{\alpha \beta}}{4}\left(F_{\gamma \nu} H_{\nu \gamma}\right)_{, \beta} \\
& =F_{\alpha \gamma, \beta} H_{\gamma \beta}+\frac{4 \pi}{c} F_{\alpha \gamma} j_{\gamma}-\frac{1}{4}\left(F_{\gamma \nu} H_{\nu \gamma}\right)_{, \alpha} .
\end{aligned}
$$

Here we have taken advantage of nonhomogeneous Maxwell equations (2.4). Further we transform the first term on the right-hand side in the last equality in (4.11)

$$
\begin{aligned}
F_{\alpha \gamma, \beta} H_{\gamma \beta} & =\frac{1}{2}\left(F_{\alpha \gamma, \beta} H_{\gamma \beta}+F_{\alpha \beta, \gamma} H_{\beta \gamma}\right) \\
& =-\frac{1}{2}\left(F_{\gamma \alpha, \beta}+F_{\alpha \beta, \gamma}\right) H_{\gamma \beta}=\frac{1}{2} F_{\beta \gamma, \alpha} H_{\gamma \beta} .
\end{aligned}
$$

When deriving the last equality in (4.12) we have used the homogeneous Maxwell equations (2.3). The substitution of (2.3) into (4.11) yields

$$
\begin{aligned}
4 \pi T_{\alpha \beta, \beta}^{\mathrm{M}} & =\frac{4 \pi}{c} F_{\alpha \gamma} j_{\gamma}+\frac{1}{2}\left(F_{\beta \gamma} H_{\gamma \beta}\right)_{, \alpha}-\frac{1}{2} F_{\beta \gamma} H_{\gamma \beta, \alpha}-\frac{1}{4}\left(F_{\gamma \nu} H_{\nu \gamma}\right)_{, \alpha} \\
& =\frac{4 \pi}{c} F_{\alpha \gamma} j_{\gamma}+\frac{1}{4}\left(F_{\beta \gamma} H_{\gamma \beta}\right)_{, \alpha}-\frac{1}{2} F_{\beta \gamma} H_{\gamma \beta, \alpha} \\
& =\frac{4 \pi}{c} F_{\alpha \gamma} j_{\gamma}+\frac{1}{4}\left(F_{\beta \gamma, \alpha} H_{\gamma \beta}-F_{\beta \gamma} H_{\gamma \beta, \alpha}\right) .
\end{aligned}
$$

In another way formula (4.13) was derived in Ref. [9, p. 43].

Now we transform the expression in round brackets on the right-hand side of Eq. (4.13) by making use of the Minkowski constitutive relations (2.18), (2.19), i.e., on $\bar{\Gamma}$. The definitions (2.12) and (2.13) can be inverted, expressing the tensors $F_{\mu \nu}$, and $H_{\mu \nu}$ in terms of $E_{\mu}, B_{\mu}, D_{\mu}, H_{\mu}$ and $u_{\mu}$ :

$$
\begin{aligned}
& F_{\mu \nu}=u_{\mu} E_{\nu}-u_{\nu} E_{\mu}+\mathrm{i} \varepsilon_{\mu \nu \gamma \rho} u_{\gamma} B_{\rho} \\
& H_{\mu \nu}=u_{\mu} D_{\nu}-u_{\nu} D_{\mu}+\mathrm{i} \varepsilon_{\mu \nu \gamma \rho} u_{\gamma} H_{\rho} .
\end{aligned}
$$

These relations can be easily checked in the co-moving reference frame $K^{\prime}$, where $u_{\gamma}^{\prime}=$ $(0,0,0, i)$, and by virtue of their covariance they are valid in an arbitrary inertial reference frame $K$. 
In (4.14) and (4.15) we take into account the constitutive relations (2.18) and (2.19)

$$
\begin{aligned}
F_{\mu \nu} & =u_{\mu} E_{\nu}-u_{\nu} E_{\mu}+\mathrm{i} \mu \varepsilon_{\mu \nu \gamma \rho} u_{\gamma} H_{\rho} \\
& =\mathcal{E}_{\mu \nu}+\mathrm{i} \mu \mathcal{H}_{\mu \nu} \\
H_{\mu \nu} & =\varepsilon\left(u_{\mu} E_{\nu}-u_{\nu} E_{\mu}\right)+\mathrm{i} \varepsilon_{\mu \nu \gamma \rho} u_{\gamma} H_{\rho} \\
& =\varepsilon \mathcal{E}_{\mu \nu}+\mathrm{i} \mathcal{H}_{\mu \nu} .
\end{aligned}
$$

Here the notations are introduced

$$
\begin{gathered}
\mathcal{E}_{\mu \nu}=-\mathcal{E}_{\nu \mu}=u_{\mu} E_{\nu}-u_{\nu} E_{\mu}, \quad \mathcal{E}_{\mu \nu} \mathcal{E}_{\nu \mu}=2 E_{\nu}^{2} \\
\mathcal{H}_{\mu \nu}=-\mathcal{H}_{\nu \mu}=\varepsilon_{\mu \nu \gamma \rho} u_{\gamma} H_{\rho}, \quad \mathcal{H}_{\mu \nu} \mathcal{H}_{\nu \mu}=2 H_{\rho}^{2}, \quad \mathcal{E}_{\mu \nu} \mathcal{H}_{\nu \mu}=0 .
\end{gathered}
$$

On substituting (4.16) and (4.17) into expression in round brackets in Eq. (4.13) we get

$$
\begin{aligned}
& F_{\beta \gamma, \alpha} H_{\gamma \beta}- F_{\beta \gamma} H_{\gamma \beta, \alpha}= \\
&=\left(\mathcal{E}_{\beta \gamma}+\mathrm{i} \mu \mathcal{H}_{\beta \gamma}\right)_{, \alpha}\left(\varepsilon \mathcal{E}_{\gamma \beta}+\mathrm{i} \mathcal{H}_{\gamma \beta}\right)-\left(\mathcal{E}_{\beta \gamma}+\mathrm{i} \mu \mathcal{H}_{\beta \gamma}\right)\left(\varepsilon \mathcal{E}_{\gamma \beta}+\mathrm{i} \mathcal{H}_{\gamma \beta}\right)_{, \alpha}= \\
& \quad=-\varepsilon_{, \alpha} \mathcal{E}_{\gamma \beta} \mathcal{E}_{\beta \gamma}-\mu_{, \alpha} \mathcal{H}_{\gamma \beta} \mathcal{H}_{\beta \gamma}+N_{\alpha}=-2 \varepsilon_{, \alpha} E_{\nu}^{2}-2 \mu_{, \alpha} H_{\rho}^{2}+N_{\alpha},
\end{aligned}
$$

where the vector $N_{\alpha}$ is

$$
N_{\alpha}=\mathrm{i}(\varepsilon \mu-1)\left(\mathcal{E}_{\beta \gamma} \mathcal{H}_{\gamma \beta, \alpha}-\mathcal{E}_{\beta \gamma, \alpha} \mathcal{H}_{\gamma \beta}\right)
$$

Obviously $N_{\alpha}=0$ if the velocity $u_{\beta}$ is the same at any points of a medium, i.e., $u_{\beta, \alpha}=0$. Indeed, in this case the velocity $u_{\beta}$ in (4.20) can be taken out of the differentiation sign and each term will contain the multiplier $\varepsilon_{\mu \nu \rho \sigma} u_{\nu} u_{\rho} \ldots$ which vanishes.

Now let the medium velocity be varying in space and in time. In this case a nonvanishing contribution to (4.20) arises only when the differentiation operator $\partial_{\alpha}$ acts on the medium velocity but not on the vectors $E_{\nu}$ and $H_{\mu}$ (see definitions of $\mathcal{E}_{\mu \nu}$ and $\mathcal{H}_{\mu \nu}$ in (4.18)). Taking into account all this, one finds easily

$$
\mathcal{E}_{\beta \gamma} \mathcal{H}_{\gamma \beta, \alpha}=-2 \mathrm{i} \Omega_{\nu} u_{\nu, \alpha}, \quad \mathcal{E}_{\beta \gamma, \alpha} \mathcal{H}_{\gamma \beta}=-2 \mathrm{i} \Omega_{\nu} u_{\nu, \alpha}
$$

where $\Omega_{\nu}$ is the Minkowski vector (2.43). Substitution of (4.21) into (4.20) gives

$$
N_{\alpha}=4(\varepsilon \mu-1) \Omega_{\nu} u_{\nu, \alpha}
$$


Further simplification of Eq. (4.22) can be achieved by expressing its right-hand side in terms of spatial components of the vectors $\Omega_{\nu}$ and $u_{\nu}$ by making use of Eqs. (2.14) and (2.48). To this end we introduce the 3 -dimensional vector $\mathfrak{W}$ through the equality [3]

$$
(\varepsilon \mu-1) \Omega_{\alpha}=\left(\frac{\mathfrak{W}}{\gamma}, \frac{\mathrm{i}}{\gamma}(\mathbf{q} \mathfrak{W})\right) .
$$

In this definition the factor $\gamma^{-1}$ is introduced which is canceled in subsequent equations where $(\varepsilon \mu-1) \Omega_{\alpha}$ is multiplied by $u_{\beta}$. Now Eq. (4.22) assumes the form

$$
N_{\alpha}=4\left(\mathfrak{W} \mathfrak{q} \mathbf{q}_{\alpha}\right) .
$$

On the physical configuration space $\bar{\Gamma}$, the vector $\mathfrak{W}$ is given by (see the Appendix B)

$$
\mathfrak{W}=[\mathbf{D B}]-[\mathbf{E H}]+\gamma^{2} \mathbf{q}(\mathbf{q},[\mathbf{D B}]-[\mathbf{E H}])
$$

It is worth noting that the medium characteristics $\varepsilon$ and $\mu$ do not enter into this formula explicitly. Substituting (4.19) and (4.24) in (4.13), we obtain on $\bar{\Gamma}$ the final compact formula for the ponderomotive forces in the Minkowski approach

$$
f_{\alpha}^{\mathrm{M}} \equiv T_{\alpha \beta, \beta}^{\mathrm{M}}=\frac{1}{c} F_{\alpha \gamma} j_{\gamma}-\frac{1}{8 \pi}\left(\varepsilon_{, \alpha} E_{\nu}^{2}+\mu_{, \alpha} H_{\nu}^{2}-2\left(\mathfrak{\mathfrak { W }} \mathbf{q}_{, \alpha}\right)\right)
$$

where the Lorentz vectors $E_{\nu}$ and $H_{\nu}$ are defined in $(2.12)$ and $(2.13)$.

Let us write down separately the spatial and temporal components of the 4 -force $f_{\alpha}^{\mathrm{M}}$ defined in (4.26),

$$
\begin{aligned}
\mathbf{f}^{\mathrm{M}} & =\rho \mathbf{E}+\frac{1}{c}[\mathbf{j} \mathbf{B}]-\frac{1}{8 \pi}\left(E_{\nu}^{2} \boldsymbol{\nabla} \varepsilon+H_{\nu}^{2} \nabla \mu-2\left(\mathfrak{W}_{i} \boldsymbol{\nabla} q_{i}\right)\right), \\
\frac{c}{\mathrm{i}} f_{4}^{\mathrm{M}} & =(\mathbf{j E})+\frac{1}{8 \pi}\left(\dot{\varepsilon} E_{\nu}^{2}+\dot{\mu} H_{\nu}^{2}-2(\mathfrak{\mathfrak { W }} \dot{\mathbf{q}})\right) .
\end{aligned}
$$

Differentiation with respect to time in (4.28) is denoted, as before, by a dot. If the medium is at rest $(\mathbf{v}=0)$, then the force $\mathbf{f}^{\mathrm{M}}$ is converted into Eq. (4.3), which in turn coincides with Eq. (3.10) for the ponderomotive force exerted by a static external electromagnetic field. Let us note the peculiarities of the obtained expression (4.27) for the ponderomotive forces exerted on the medium in the Minkowski approach.

- This formula does not contain the derivatives of the field vectors $\mathbf{E}, \mathbf{H}, \mathbf{D}$, and $\mathbf{B}$.

- If the medium is homogeneous $\left(\varepsilon_{, k}=0, \mu_{, k}=0\right)$ and its velocity is the same at all medium points $\left(\mathbf{q}_{, k}=0\right)$, then the ponderomotive force vanishes. 
It should be emphasized that we succeeded in revealing these features of the force $\mathbf{f}^{\mathrm{M}}$ just because the calculation was carried out on the physical configuration space $\bar{\Gamma}$.

To make presentation complete, we write down, in an explicit form, the Lorentz force (the first term on the right-hand side of Eq. (4.26)) for special cases when there is only convection current (the respective Lorentz force is $f_{\alpha}^{\text {conv }}$ ) or only the conduction current (the Lorentz force is $\left.f_{\alpha}^{\text {cond }}\right)$.

In the first case, with allowance for Eq. (2.30), we obtain

$$
f_{\alpha}^{\text {conv }}=\frac{1}{c} F_{\alpha \gamma} j_{\gamma}^{\text {conv }}=\rho^{0} F_{\alpha \gamma} u_{\gamma}=\rho^{0} E_{\alpha}
$$

where $\rho^{0}$ is the density of convection charges in the co-moving reference frame $(2.24),(2.25)$.

In deriving the Lorentz force experienced by the conduction current we take first into account the covariant Ohm law (2.37)

$$
f_{\alpha}^{\text {cond }}=\frac{1}{c} F_{\alpha \beta} j_{\beta}^{\text {cond }}=\frac{\lambda}{c} F_{\alpha \beta} E_{\beta} .
$$

And now we take advantage of the representation (4.16) for the tensor $F_{\alpha \beta}$, which holds on $\bar{\Gamma}$,

$$
\begin{aligned}
f_{\alpha}^{\text {cond }} & =\frac{\lambda}{c}\left(u_{\alpha} E_{\beta}-u_{\beta} E_{\alpha}\right) E_{\beta}+\mathrm{i} \mu \frac{\lambda}{c} \varepsilon_{\alpha \beta \gamma \delta} u_{\gamma} H_{\delta} E_{\beta} \\
& =\frac{\lambda}{c} u_{\alpha} E_{\gamma}^{2}+\mu \frac{\lambda}{c} \Omega_{\alpha},
\end{aligned}
$$

where $\Omega_{\alpha}$ is the Minkowski vector (2.43). In the rest frame we have

$$
\left(\mathbf{f}^{\text {cond }}\right)^{\prime}=\mu \frac{\lambda}{c}\left[\mathbf{E}^{\prime} \mathbf{H}^{\prime}\right], \quad\left(f_{4}^{\text {cond }}\right)^{\prime}=\mathrm{i} \frac{\lambda}{c} \mathbf{E}^{\prime 2} .
$$

\section{DIFFERENT REPRESENTATIONS OF THE ABRAHAM ENERGY-MOMENTUM TENSOR}

The calculation of the ponderomotive forces in the framework of the Abraham approach is simplified essentially if a special representation of the Abraham energy-momentum tensor is employed. Let us consider briefly different representations for this tensor [3].

Abraham was guided by the symmetry requirement imposed on the energy-momentum tensor in a medium. He has constructed his tensor taking into account the Minkowski constitutive relations, i.e., on $\bar{\Gamma}[10] 12]$. 
Considerably later the explicitly covariant representation for the symmetric energymomentum tensor was found which holds on the whole configuration space $\Gamma$ [25], [5], [6, 7], [3]. The equivalence on $\bar{\Gamma}$ of the covariant representation and the original 3-dimensional Abraham formulae was proved in Ref. [3].

Here we derive the original Abraham formulae, which will be used further, proceeding from the covariant representation for the energy-momentum tensor. The Abraham derivation of the energy-momentum tensor is traced in detail in Ref. [3] .

The explicitly covariant symmetric energy-momentum tensor of electromagnetic field in a medium $T_{\alpha \beta}^{\mathrm{sym}}$ is defined by the formulae [3]:

$$
T_{\alpha \beta}^{\mathrm{sym}}=\frac{1}{2}\left(T_{\alpha \beta}^{\mathrm{M}}+T_{\beta \alpha}^{\mathrm{M}}\right)+A_{\alpha \beta},
$$

where

$$
\begin{gathered}
A_{\alpha \beta}=A_{\beta \alpha}=\frac{1}{8 \pi}\left\{u_{\alpha}\left(\widetilde{\Omega}_{\beta}-\Omega_{\beta}\right)+u_{\beta}\left(\widetilde{\Omega}_{\alpha}-\Omega_{\alpha}\right)\right\} \\
=\frac{1}{8 \pi}\left\{u_{\alpha}\left(F_{\beta \nu} H_{\nu \lambda} u_{\lambda}-H_{\beta \nu} F_{\nu \lambda} u_{\lambda}\right)+u_{\beta}\left(F_{\alpha \nu} H_{\nu \lambda} u_{\lambda}-H_{\alpha \nu} F_{\nu \lambda} u_{\lambda}\right)\right\} .
\end{gathered}
$$

Here $\Omega_{\alpha}$ and $\widetilde{\Omega}_{\beta}$ are the Minkowski vectors (2.43) and (2.46), respectively. By making use of the values of these vectors in the co-moving reference frame $K^{\prime}$ (Eqs. (2.44) and (2.47)) one can easily show that the tensor (5.1)

$$
T_{\alpha \beta}^{\mathrm{sym}}=\left(\begin{array}{cc}
\sigma_{i j}^{\mathrm{sym}} & -\mathrm{i} c \mathbf{g}^{\mathrm{sym}} \\
-\frac{\mathrm{i}}{c} \mathbf{S}^{\mathrm{sym}} & w^{\mathrm{sym}}
\end{array}\right)
$$

in $K^{\prime}$ has the components

$$
\begin{gathered}
\sigma_{i j}^{\prime}=\frac{1}{2}\left(\sigma_{i j}^{\prime \mathrm{M}}+\sigma_{j i}^{\prime \mathrm{M}}\right) \\
\frac{1}{c} \mathbf{S}^{\prime \mathrm{sym}}=c \mathbf{g}^{\prime \mathrm{sym}}=\frac{1}{c} \mathbf{S}^{\prime \mathrm{P}}=\frac{1}{4 \pi}\left[\mathbf{E}^{\prime} \mathbf{H}^{\prime}\right] \\
w^{\prime \mathrm{sym}}=w^{\prime \mathrm{M}}=\frac{1}{8 \pi}\left(\mathbf{E}^{\prime} \mathbf{D}^{\prime}+\mathbf{H}^{\prime} \mathbf{B}^{\prime}\right) .
\end{gathered}
$$

It will be recalled that we mark by prime the quantities in the co-moving reference frame $K^{\prime}$. Conditions (5.5)-(5.7) are satisfied without invoking constitutive relations (2.10). It is this point that distinguish the tensor (5.1) from the energy-momentum tensor constructed by Abraham (see further and Ref. [3]). Conditions (5.5)-(15.7) specify the tensor $T_{\alpha \beta}^{\mathrm{sym}}$ uniquely.

Equations (5.1) - (5.3) were derived in our paper [3] by generalization of the Abraham reasoning. Now, going in the opposite direction, we show how to obtain the Abraham 
formulae proceeding from the covariant representation (5.1)-(5.3). In this consideration Eq. (5.2) plays a key role (this formula was derived in Ref. [3] ).

The tensor $T_{\alpha \beta}^{\mathrm{sym}}($ (5.1) is defined on the whole configuration space $\Gamma$ while Abraham has derived his formulae with allowance for the constitutive relations, i.e., on $\bar{\Gamma}$. Thus we have to take into account the Minkowski material relations (2.18), (2.19) in Eqs. (5.1)-(5.3). It is worth noting that Abraham used the Minkowski constitutive relations specifically. Namely, they were not employed to express one pair of field vectors $\mathbf{E}, \mathbf{H}, \mathbf{D}$, and $\mathbf{B}$ in terms of the rest pair, but a special formulae were used which contain all the vectors $\mathbf{E}, \mathbf{H}, \mathbf{D}, \mathbf{B}$, v, and the material characteristics of the medium $\varepsilon$ and $\mu$, these formulae being valid only under fulfilment of the Minkowski constitutive relations, i.e., on $\bar{\Gamma}$. An important example of such formulae is the equality (B1), proved in the Appendix B, and the following relations which is also valid on $\bar{\Gamma}$ :

$$
\widetilde{\Omega}_{\alpha}-\Omega_{\alpha}=(\varepsilon \mu-1) \Omega_{\alpha}
$$

Equation (15.8) can be easily derived if we substitute in the definition of $\widetilde{\Omega}_{\alpha}$ (Eq. (2.46) $)$ the constitutive relations in the form (2.18), (2.19) and take into account the definition of $\Omega_{\alpha}$ in Eq. (2.43). As a result the tensor (5.1), (5.2) reduces on $\bar{\Gamma}$ to the Abraham energymomentum tensor [3]]:

$$
T_{\alpha \beta}^{\mathrm{A} 1}=\frac{1}{2}\left(T_{\alpha \beta}^{\mathrm{M}}+T_{\alpha \beta}^{\mathrm{M}}\right)+\frac{\varepsilon \mu-1}{8 \pi}\left(u_{\alpha} \Omega_{\beta}-u_{\beta} \Omega_{\alpha}\right) .
$$

The Abraham tensor $T_{\alpha \beta}^{\mathrm{A} 1}$ in the co-moving reference frame $K^{\prime}$ satisfies conditions (5.5)(5.7) only if the constitutive relations (2.10) are met, i.e., on $\bar{\Gamma}$. Therefore these conditions do not fix this tensor uniquely and it can be represented on $\bar{\Gamma}$ in different forms. To show this we take advantage of the equality (B1), proved in Appendix B. Let us write down the tensor $T_{\alpha \beta}^{\mathrm{A} 1}$ and the equality (B1), multiplied by $1 / 2$, on the neighbouring lines:

$$
\begin{aligned}
& 4 \pi T_{\alpha \beta}^{\mathrm{A} 1}=\frac{1}{2} F_{\alpha \gamma} H_{\gamma \beta}+\frac{1}{2} F_{\beta \gamma} H_{\gamma \alpha}-\frac{1}{4} \delta_{\alpha \beta} F_{\gamma \delta} H_{\delta \gamma}+\frac{\varepsilon \mu-1}{2}\left(u_{\alpha} \Omega_{\beta}+u_{\beta} \Omega_{\alpha}\right), \\
& 0=\frac{1}{2} F_{\alpha \gamma} H_{\gamma \beta}-\frac{1}{2} F_{\beta \gamma} H_{\gamma \alpha} \quad-\quad \frac{\varepsilon \mu-1}{2}\left(u_{\alpha} \Omega_{\beta}-u_{\beta} \Omega_{\alpha}\right) \text {. }
\end{aligned}
$$

Adding and subtracting the left-hand sides and the right-hand sides of these equations we obtain on $\bar{\Gamma}$ :

$$
T_{\alpha \beta}^{\mathrm{A} 1}=T_{\alpha \beta}^{\mathrm{A} 2}=T_{\alpha \beta}^{\mathrm{A} 3},
$$


where

$$
\begin{aligned}
& T_{\alpha \beta}^{\mathrm{A} 2}=T_{\alpha \beta}^{\mathrm{M}}+\frac{\varepsilon \mu-1}{4 \pi} u_{\beta} \Omega_{\alpha}, \\
& T_{\alpha \beta}^{\mathrm{A} 3}=T_{\beta \alpha}^{\mathrm{M}}+\frac{\varepsilon \mu-1}{4 \pi} u_{\alpha} \Omega_{\beta} .
\end{aligned}
$$

Following in this way, one can also obtain other representations for the Abraham energymomentum tensor on $\bar{\Gamma}[3]$. The introduction of the vector $\mathfrak{W}$ (Eq. (4.25)) through the substitution (4.23) removes the explicit dependence on $\varepsilon \mu$ in tensors $T_{\alpha \beta}^{\mathrm{A} i}, i=1,2,3$. By making use of the vector $\mathfrak{W}$, Abraham derived explicit formulae, in terms of the 3-dimensional vectors, for all the components of the tensors (15.9) and (5.13) (see respectively Refs. [12] and [10] as well as our paper [3]). However we shall not use these 3-dimensional vector formulae further. In calculation of the ponderomotive forces in the Abraham approach we will employ Eq. (5.13).

It is worthy to note that Eqs. (5.9), (15.13), and (5.14) for the Abraham tensor were constructed by Grammel [26]. Possibility of representing the Abraham tensor in different forms, not all of them being explicitly symmetric (see Eqs. (5.13) and (5.14)), is explained by a restricted domain of their validity, namely, they hold only on $\bar{\Gamma}$.

\section{PONDEROMOTIVE FORCES IN THE ABRAHAM APPROACH}

At the outset we envisage the medium at rest and take advantage of Eq. (3.18). Following the requirement of the symmetry of the energy-momentum tensor, Abraham defined (in fact postulated) the momentum density of electromagnetic field in a medium in the form

$$
\mathbf{g}^{\mathrm{A}}=\frac{1}{4 \pi c}[\mathbf{E H}]
$$

(see Eq. (5.6)). As a result, the additional term (3.14) in Eq. (3.18) is not canceled and we get

$$
\begin{aligned}
\left.\mathbf{f}^{\mathrm{A}}\right|_{\mathbf{v}=0} & =\boldsymbol{\sigma}-\mathbf{g}^{\mathrm{A}} \\
& =\left.\mathbf{f}^{\mathrm{M}}\right|_{\mathbf{v}=0}+\frac{1}{4 \pi c} \frac{\partial}{\partial t}([\mathbf{D B}]-[\mathbf{E H}]) .
\end{aligned}
$$

The last term in this formula is the Abraham force $[13, \S 75]$. We introduce a special notation for this term

$$
\left.\Delta \mathbf{f}^{\mathrm{A}}\right|_{\mathbf{v}=0}=\frac{1}{4 \pi c} \frac{\partial}{\partial t}([\mathbf{D B}]-[\mathbf{E H}]),
$$


in oder to distinguish this force from the total ponderomotive force $\mathbf{f}^{\mathrm{A}}$ in the Abraham approach.

Now we turn to the derivation of the covariant formula for the ponderomotive 4 -force in the Abraham approach in the case of moving medium. Abraham, just as Minkowski, lets the ponderomotive force to be equal to the divergence of the energy-momentum tensor of electromagnetic field in a medium. For our consideration it is crucial that the Abraham energy-momentum tensor can be represented on $\bar{\Gamma}$ in different forms (see preceding Section $\mathrm{V})$. We shall use the form of this tensor which is the most close, in construction, to the Minkowski energy-momentum tensor. In the preceding Section this representation for the Abraham tensor was denoted by $T_{\alpha \beta}^{\mathrm{A} 2}$ (see Eq. (5.13) $)$,

$$
T_{\alpha \beta}^{\mathrm{A} 2}=T_{\alpha \beta}^{\mathrm{M}}+\frac{\varepsilon \mu-1}{4 \pi} u_{\beta} \Omega_{\alpha},
$$

where $T_{\alpha \beta}^{\mathrm{M}}$ is the Minkowski energy-momentum tensor (4.4), $\Omega_{\alpha}$ is the Minkowski vector (2.43), and $u_{\alpha}$ is the 4 -velocity of the medium (2.14). The right-hand-side of Eq. (6.4) is not symmetric with respect to indices $\alpha \beta$, however it was shown in Sec. V that on $\bar{\Gamma}$ tensor $T_{\alpha \beta}^{\mathrm{A} 2}$ coincides with explicitly symmetric tensor $T_{\alpha \beta}^{\mathrm{A} 1}$ (5.9) (see Eq. (5.12)).

Differentiation of Eq. (6.4) gives the ponderomotive force in the Abraham approach

$$
f_{\alpha}^{\mathrm{A}} \equiv T_{\alpha \beta, \beta}^{\mathrm{A} 2}=f_{\alpha}^{\mathrm{M}}+\frac{1}{4 \pi}\left\{(\varepsilon \mu-1) \Omega_{\alpha} u_{\beta}\right\}_{, \beta},
$$

where $f_{\alpha}^{\mathrm{M}}$ is the ponderomotive force in the Minkowski approach (4.26).

Thus it is required to calculate only the difference between ponderomotive forces in the Abraham and Minkowski approaches

$$
\Delta f_{\alpha} \equiv f_{\alpha}^{\mathrm{A}}-f_{\alpha}^{\mathrm{M}}=\frac{1}{4 \pi}\left\{(\varepsilon \mu-1) \Omega_{\alpha} u_{\beta}\right\}_{, \beta}
$$

In the rest frame $K^{\prime}$ we have

$$
\begin{aligned}
\Delta \mathbf{f}^{\prime} & =\frac{1}{4 \pi c} \frac{\partial}{\partial t^{\prime}}\left\{(\varepsilon \mu-1)\left[\mathbf{E}^{\prime} \mathbf{H}^{\prime}\right]\right\} \\
\Delta f_{4}^{\prime} & =0
\end{aligned}
$$

Upon taking into account the constitutive relations (2.10) in Eq. (6.3), we see that Eqs. (6.7) and (6.3) coincide on $\bar{\Gamma}$. 
The right-hand side of Eq. (6.6) is expressed in terms of two 3-dimensional vectors: the medium velocity $\mathbf{q}=\mathbf{v} / c$ and the vector $\mathfrak{W}$ introduced in (4.23),

$$
\begin{aligned}
4 \pi \Delta \mathbf{f} & =(\mathbf{q} \boldsymbol{\nabla}) \mathfrak{W}+\mathfrak{W} \operatorname{div} \mathbf{q}+\frac{1}{c} \mathfrak{W}_{, t}, \\
\frac{4 \pi}{\mathrm{i}} \Delta f_{4} & =(\mathbf{q} \boldsymbol{\nabla})(\mathbf{q} \mathfrak{W})+(\mathbf{q} \mathfrak{W}) \operatorname{div} \mathbf{q}+\frac{1}{c}(\mathbf{q} \mathfrak{W})_{, t} .
\end{aligned}
$$

Let us remind that the vector $\mathfrak{W}$ is defined on $\bar{\Gamma}$ by Eq. (4.25) and does not depend explicitly on the medium characteristics $\varepsilon$ and $\mu$.

Equations (6.6) and (6.9), (6.10) together with Eqs. (4.26), (4.27), and (4.28) provide a complete solution of the problem about the calculation of the ponderomotive force in the Abraham approach. In order to represent the covariant formulae (6.6) and (4.26) in terms of the same variables it is sufficient to substitute the vector $N_{\alpha}$ in the form (4.22) into Eq. (4.26). It is worth noting that Eqs. (6.5)-(6.10) are derived on the physical configuration space $\bar{\Gamma}$. It is this circumstance that enables us to obtain compact and convenient to analyse formulae determining the ponderomotive forces in the Minkowski and Abraham approaches.

\section{CONCLUSION}

In the general setting of the problem, the explicit compact formulae are derived for the ponderomotive forces in the Minkowski approach (Eqs. (4.26)-(4.28)) and in the Abraham approach (Eqs. (6.5)-(6.10) ). Two principal points in calculation should be stressed: i) the formulae are derived on the physical configuration space $\bar{\Gamma}$, i.e., with allowance for the the Minkowski constitutive relations; ii) a special representation for the Abraham energymomentum tensor (6.4) is used which is the most close, in construction, to the Minkowski tensor. In deriving the ponderomotive forces the most general case is considered, namely, nonhomogeneous nonstationary medium, the external electromagnetic field and medium velocity can vary in space and with time.

The formulae obtained (see Eqs. (6.6) -(6.10) ) give a simple expression for the difference of the ponderomotive forces in the approaches under consideration. Thus we have derived the generalization to the moving media of the known expression for the Abraham force known for media at rest $[13, \S 75]$.

An interesting result obtained in this paper is also the derivation of the Lorentz force which is exerted by external electromagnetic field on the conduction current in a medium. 
In deriving the formulae (4.31) and (4.32) for this force the covariant Ohm law and the constitutive Minkowski relations were taken into account.

\section{Appendix A: Ponderomotive forces in vacuum}

The electric charges (with density $\rho$ ) and currents (with density $\mathbf{j}$ ), placed in vacuum, experience the Lorentz force

$$
f_{\mu}=\frac{1}{c} F_{\mu \nu} j_{\nu}
$$

from electromagnetic field $F_{\mu \nu}$ created by these charges and currents. In Eq. (A1) $j_{\nu}=(\mathbf{j}, \mathrm{i} c \rho)$ and tensor $F_{\mu \nu}$ is obtained from (2.5) by substituting $H_{k}$ in place of $B_{k}$. All the charges and currents we treat on the same footing without dividing them into those involved in the system under consideration and external ones with respect to this system. In addition, we digress from their self-action [8, sec. 42], [5, Chap. III].

Taking into account the Maxwell equations in vacuum

$$
\begin{gathered}
F_{\alpha \beta, \gamma}+F_{\beta \gamma, \alpha}+F_{\gamma \alpha, \beta}=0, \\
F_{\alpha \beta, \beta}=\frac{4 \pi}{c} j_{\alpha},
\end{gathered}
$$

we can represent the Lorentz force (A1) as the 4-divergence of the energy-momentum tensor of electromagnetic field in vacuum [14, 24]

$$
f_{\mu}=T_{\mu \nu, \nu}
$$

where

$$
T_{\mu \nu}=\frac{1}{4 \pi}\left(F_{\mu \lambda} F_{\lambda \nu}-\frac{\delta_{\mu \nu}}{4} F_{\lambda \rho} F_{\rho \lambda}\right) .
$$

Let us prove this assertion. Substitution of (A5) into (A4) gives

$$
4 \pi f_{\mu}=\left(F_{\mu \lambda} F_{\lambda \nu}\right)_{, \nu}-\frac{1}{4}\left(F_{\lambda \rho} F_{\rho \lambda}\right)_{, \mu} .
$$

The first term on the right-hand side of (A6) we transform by making use of Maxwell's equations (A2) and (A3)

$$
\begin{aligned}
\left(F_{\mu \lambda} F_{\lambda \nu}\right)_{, \nu}=F_{\mu \lambda} F_{\lambda \nu, \nu}+F_{\mu \lambda, \nu} & F_{\lambda \nu} \\
= & \frac{4 \pi}{c} F_{\mu \lambda} j_{\lambda}+\frac{1}{2}\left(F_{\mu \lambda, \nu}-F_{\mu \nu, \lambda}\right) F_{\lambda \nu} \\
& =\frac{4 \pi}{c} F_{\mu \lambda, j_{\lambda}}-\frac{1}{2} F_{\lambda \nu, \mu} F_{\lambda \nu}=\frac{4 \pi}{c} F_{\mu \lambda, j}+\frac{1}{4}\left(F_{\lambda \nu} F_{\nu \lambda}\right)_{, \mu}
\end{aligned}
$$


Substitution of (A7) in (A6) results in the Lorentz formula (A1)

$$
f_{\mu}=T_{\mu \nu, \nu}=\frac{1}{c} F_{\mu \lambda} j_{\lambda}
$$

These equalities can be interpreted in the following way. Two expressions for the Lorentz force presented in (A8) are equivalent with allowance for Maxwell's equations (A2), (A3). Let us write down the components of these expressions for the Lorentz force. To this end it is convenient to use the standard notations for the individual components of the energymomentum tensor $T_{\mu \nu}(\underline{\mathrm{A} 5})$ :

$$
T_{\mu \nu}=\left(\begin{array}{cc}
\sigma_{i j} & -\mathrm{i} c \mathbf{g} \\
-\frac{\mathrm{i}}{c} \mathbf{S}^{\mathrm{P}} & w
\end{array}\right),
$$

where

$$
\begin{gathered}
\sigma_{i j}=\frac{1}{4 \pi}\left\{E_{i} E_{j}+H_{i} H_{j}-\frac{\delta_{i j}}{2}(\mathbf{E E}+\mathbf{H H})\right\}, \\
\frac{1}{c} \mathbf{S}^{\mathrm{P}}=\frac{c}{4 \pi}[\mathbf{E H}], \quad c \mathbf{g}=\frac{1}{c} \mathbf{S}^{\mathrm{P}}, \\
w=\frac{1}{8 \pi}(\mathbf{E E}+\mathbf{H H}) .
\end{gathered}
$$

Here $\mathbf{S}^{\mathrm{P}}$ is the Poynting vector (2.42).

On substituting (A9) in (A8), we get

$$
\begin{aligned}
\mathbf{f} & =\boldsymbol{\sigma}-\dot{\mathbf{g}}=\rho \mathbf{E}+\frac{1}{c}[\mathbf{j} \mathbf{H}], \\
f_{4} & =-\frac{\mathrm{i}}{c}\left(\operatorname{div} \mathbf{S}^{\mathrm{P}}+\dot{w}\right)=\frac{\mathrm{i}}{c}(\mathbf{j E}),
\end{aligned}
$$

where $\boldsymbol{\sigma} \equiv\left(\sigma_{1}, \sigma_{2}, \sigma_{3}\right), \quad \sigma_{i}=\sigma_{i k, k}$. For brevity the subscript vac is omitted in this Appendix.

The foregoing reasoning devoted to the derivation of Eq. (A8), proceeding from the given form of the energy-momentum tensor (A5), can be inverted, namely, one can find the energy-momentum tensor (A5), requiring the fulfilment of the second equality in Eq. (A8) with allowance of the Maxwell equations. Let us prove this assertion. We replace in (A8) the current $j_{\lambda}$ by making use of nonhomogeneous Maxwell's equations (A3):

$$
\begin{aligned}
T_{\mu \nu, \nu} & =\frac{1}{c} F_{\mu \lambda} \frac{c}{4 \pi} F_{\lambda \rho, \rho} \\
& =\frac{1}{4 \pi}\left(F_{\mu \lambda} F_{\lambda \rho}\right)_{, \rho}-\frac{1}{4 \pi} F_{\mu \lambda, \rho} F_{\lambda \rho} .
\end{aligned}
$$


Further with the help of homogeneous Maxwell's equations (A2) we can write

$$
-F_{\mu \lambda, \rho} F_{\lambda \rho}=-\frac{1}{2}\left(F_{\mu \lambda, \rho}+F_{\rho \mu, \lambda}\right) F_{\lambda \rho}=\frac{1}{2} F_{\lambda \rho, \mu} F_{\lambda \rho}
$$

From (A13) and (A14) it follows

$$
4 \pi T_{\mu \nu, \nu}=\left(F_{\mu \lambda} F_{\lambda \nu}\right)_{, \nu}-\frac{1}{4}\left(F_{\lambda \rho} F_{\rho \lambda}\right)_{, \mu}
$$

Obviously tensor $T_{\mu \nu}$ in the form (A5) satisfies Eq. (A17).

Thus one can conclude that the expression for the Lorentz force (A1) and the explicit form of the energy-momentum tensor of the electromagnetic field in vacuum (A5) (the Lorentz theory of electrons) are equivalent in the meaning specified above.

In the macroscopic electrodynamics (in the theory of electromagnetic field in a medium) the situation is essentially different. As was noted in Section III, it does not turn out well to obtain the expression for the ponderomotive forces exerting on the medium (the analogue of Eq. (A1) ) proceeding from the Lorentz theory of electrons. The analogy with the electromagnetic field in vacuum can provide only the definition of the ponderomotive forces through the energy-momentum tensor (A4). This ensures the covariance of the definition and reduces the problem to looking for the macroscopic energy-momentum tensor of electromagnetic field.

\section{Appendix B: The explicit formula for vector $\mathfrak{W}$}

In order to find the explicit expression for the vector $\mathfrak{W}$ on $\bar{\Gamma}$ (see Eq. (4.23)) we take advantage of the equality valid on $\bar{\Gamma}[15$, p. 51, Eq. (119)]:

$$
\left.(\varepsilon \mu-1)\left(u_{\alpha} \Omega_{\beta}-u_{\beta} \Omega_{\alpha}\right)\right|_{\bar{\Gamma}}=F_{\alpha \gamma} H_{\gamma \beta}-\left.F_{\beta \gamma} H_{\gamma \alpha}\right|_{\bar{\Gamma}} .
$$

First we prove this equality by the method different from that used in Ref. [15], namely, we demonstrate that on $\bar{\Gamma}$ the right-hand side of (B1) transforms into the left-hand side of this formula. To this end we employ the representations (4.16) and (4.17) for the tensors $F_{\alpha \beta}$ and $H_{\alpha \beta}$ which are valid on $\bar{\Gamma}$ :

$$
F_{\alpha \beta}=\mathcal{E}_{\alpha \beta}+\mathrm{i} \mu \mathcal{H}_{\alpha \beta}, \quad H_{\alpha \beta}=\varepsilon \mathcal{E}_{\alpha \beta}+\mathrm{i} \mathcal{H}_{\alpha \beta},
$$


where $\mathcal{E}_{\mu \nu}$ and $\mathcal{H}_{\mu \nu}$ are determined in (4.18). Substitution of (B2) into the right-hand side of (B1) yields

$$
\begin{aligned}
& F_{\alpha \gamma} H_{\gamma \beta}-\left.F_{\beta \gamma} H_{\gamma \alpha}\right|_{\Gamma}=\left(\mathcal{E}_{\alpha \gamma}+\mathrm{i} \mu \mathcal{H}_{\alpha \gamma}\right)\left(\varepsilon \mathcal{E}_{\gamma \beta}+\mathrm{i} \mathcal{H}_{\gamma \beta}\right)-(\alpha \leftrightarrow \beta) \\
& =\varepsilon \mathcal{E}_{\alpha \gamma} \mathcal{E}_{\gamma \beta}-\mu \mathcal{H}_{\alpha \gamma} \mathcal{H}_{\gamma \beta}+\mathrm{i} \varepsilon \mu \mathcal{H}_{\alpha \gamma} \mathcal{E}_{\gamma \beta}+\mathrm{i} \mathcal{E}_{\alpha \gamma} \mathcal{H}_{\gamma \beta}-(\alpha \leftrightarrow \beta) .
\end{aligned}
$$

Two first terms in the right-hand side of (B33), which are symmetric with respect to indexes $\alpha, \beta$, are canceled with the analogous terms in the expression denoted by $(\alpha \leftrightarrow \beta)$. Therefore it needs to calculate explicitly only the product $\mathcal{E}_{\alpha \gamma} \mathcal{H}_{\gamma \beta}$. With the help of the definitions (4.18), we obtain

$$
\begin{aligned}
\mathcal{E}_{\alpha \gamma} \mathcal{H}_{\gamma \beta} & =\left(u_{\alpha} E_{\gamma}-u_{\gamma} E_{\alpha}\right) \varepsilon_{\gamma \beta \delta \rho} u_{\delta} H_{\rho} \\
& =u_{\alpha} \varepsilon_{\beta \gamma \rho \delta} E_{\gamma} H_{\rho} u_{\delta}=\mathrm{i} u_{\alpha} \Omega_{\beta},
\end{aligned}
$$

where $\Omega_{\beta}$ is the Minkowski vector (2.43). Substitution of (B4) in (B3) gives

$$
\begin{aligned}
F_{\alpha \gamma} H_{\gamma \beta}-\left.F_{\beta \gamma} H_{\gamma \alpha}\right|_{\bar{\Gamma}} & =-u_{\alpha} \Omega_{\beta}+u_{\beta} \Omega_{\alpha}-\varepsilon \mu u_{\beta} \Omega_{\alpha}+\varepsilon \mu u_{\alpha} \Omega_{\beta} \\
& =\left.(\varepsilon \mu-1)\left(u_{\alpha} \Omega_{\beta}-u_{\beta} \Omega_{\alpha}\right)\right|_{\bar{\Gamma}}
\end{aligned}
$$

Thus the equality (B1) is proved.

The components of the antisymmetric tensors in the left-hand side and in the right-hand side of Eq. (B1) are determined by two 3-dimensional vectors (see notations introduced in (2.5) -(2.7) ). Therefore Eq. (B1) is equivalent to two 3-dimensional vector equalities. We write down these equalities in an explicit form by making use of Eq. (2.14) and definition (4.23).

When the pair of indexes $\alpha \beta$ in Eq. (B1) takes the values 14, 24,34 the following vector equality arises here:

$$
-\mathrm{i}\{\mathfrak{W}-\mathbf{q}(\mathbf{q} \mathfrak{W})\}=-\mathrm{i}\{[\mathbf{D B}]-[\mathbf{E H}]\}
$$

or in another form

$$
\mathfrak{W}=[\mathbf{D B}]-[\mathbf{E H}]+\mathbf{q}(\mathbf{q} \mathfrak{W})
$$

From Eq. (B7) it folows

$$
\begin{aligned}
(\mathbf{q} \mathfrak{W}) & =(\mathbf{q},[\mathbf{D B}]-[\mathbf{E H}])+q^{2}(\mathbf{q} \mathfrak{W})= \\
& =\gamma^{2}(\mathbf{q},[\mathbf{D B}]-[\mathbf{E H}]) .
\end{aligned}
$$


Finally we arrive at the result

$$
\mathfrak{W}=[\mathbf{D B}]-[\mathbf{E H}]+\gamma^{2} \mathbf{q}(\mathbf{q},[\mathbf{D B}]-[\mathbf{E H}]) .
$$

If in Eq. (B1) the pair of indexes $\alpha \beta$ assumes the values 23, 31, 12, then we obtain another vector equality

$$
[\mathbf{q} \mathfrak{W}]=[\mathbf{E D}]+[\mathbf{H B}]
$$

Substitution of (B91) into (B10) yields

$$
[\mathbf{q},[\mathbf{D B}]-[\mathbf{E H}]]=[\mathbf{E D}]+[\mathbf{H B}]
$$

We stress once more that the obtained equations (B9)-(B11), as well as the initial equality (B1), are valid only under fulfilment of the Minkowski relations, i.e., only on $\bar{\Gamma}$.

[1] I. Brevik, Mat. Phys. Medd. Dan. Vid. Selsk. 37(11), 1 (1970); 37(13), 1 (1970).

[2] I. Brevik, Phys. Rep. 52, 133 (1979).

[3] V. V. Nesterenko and A. V. Nesterenko, J. Math. Phys. 57, 032901 (2016).

[4] D. F. Nelson, Phys. Rev. A 44, 3985 (1991).

[5] S.R. de Groot and L.G. Suttorp, Foundations of Electrodynamics (North-Holland, Amstredam, 1972), Chap. V, $\S 7$.

[6] Yu. N. Obukhov, Ann. Phys. (Berlin), 17, No. 9/10, 830 (2008).

[7] V. P. Makarov and A. A. Rukhadze, Phys. Usp. 52, 937 (2011) [Usp. Fiz. Nauk 181, 1357 (2011)].

[8] W. Pauli, Relativitätstheorie (Teubner, Leipzig, 1921) [W. Pauli, Theory of Relativity (Pergamon Press, New York, 1958)].

[9] H. Minkowski, Zwei Abhandlungen über die Grundgleichungen der Elektrodynamik (B.G. Teubner, Leipzig und Berlin, 1910).

[10] M. Abraham, Theorie der Elektrizität, Bd. 2 (4. Aufl., Teubner, Leipzig, 1920).

[11] M. Abraham, Palermo Rend. 28, 1 (1909) http://en.wikisource.org/wiki/Author:Max_Abraham.

[12] M. Abraham, Palermo Rend. 30, 33 (1910) [http://en.wikisource.org/wiki/Author:Max_Abraham].

[13] L. D. Landau, E. M. Lifshitz, Electrodynamics of Continuous Media (Pergamon Press, Oxford, 1984). 
[14] L. D. Landau, E. M. Lifshitz, The Classical Theory of Fields (Pergamon Press, Oxford, 1980).

[15] H. Kafka, Ann. Physik 58, 1 (1919).

[16] Max von Laue, Das Relativitätstheorie. Erster Band. Das Relativitätsprinzip der Lorentztransformation. Vierte vermehrte Auflage (Friedr. Vieweg \& Sohn, Braunschweig, 1921).

[17] R. Becker, Theorie der Elektrizität. Band 2: Elektronentheorie (Teubner, Berlin-Leipzig, 1933).

[18] Chap. Møller, The theory of relativity (Clarendon Press, Oxford, 1972).

[19] G. Herglotz, Ann. Physik 36, 497 (1911).

[20] Yu. V. Novozhilov, Yu. A. Yappa, Electrodynamics (Mir Publishers, Moscow, Russia, 1986).

[21] J. A. Stratton, Electromagnetic Theory (Adams Press, Chicago, Illinois, USA, 2008).

[22] W. K. H. Panofsky, M. Phillips, Classical Electricity and Magnetism, 2nd ed. (Dover Books on Physics, 2005).

[23] I. E. Tamm, Fundamentals of the Theory of Electricity (Mir Publishers, Moscow, Russia, 1979).

[24] J. D. Jackson, Classical Electrodynamics, 3rd edn (Wiley, New York, 1998).

[25] E. Schmutzer, Relativistische Physik (Akademie Verlag, Leipzig, 1968) p. 408.

[26] R. Grammel, Ann. Physik 346, 570 (1913). 